\title{
PARÁMETROS DE CALIDAD DE CONCENTRADOS PLAQUETARIOS OBTENIDOS POR BUFFY COAT EN UN HOSPITAL GENERAL DEL PERÚ.
}

\section{QUALITY PARAMETERS OF PLATELET CONCENTRATES OBTAINED BY BUFFY COAT IN A GENERAL HOSPITAL OF PERU.}

Córdova-Vicerrel Rocío Del Rosario', Huertas-Talavera Eric ${ }^{2}$.

1. Médico Cirujano del Hospital Regional de Ica

2. Docente de la Facultad de Medicina de la Universidad Nacional "San Luis Gonzaga" de Ica

DOI: https://doi.org/10.35563/rmp.v8i3.192

\section{Correspondencia:}

Córdova-Vicerrel Rocío Del

Rosario.

Dirección: Urb. Las Palmeras L-6IV etapa Ica, Perú.

Número de celular: 948520895

Correo Electrónico:

rociocovi59@gmail.com

\section{Contribuciones De Autoría:}

CVR y HTE han participado en la concepción, diseño del artículo, análisis e interpretación de resultados. GTI participaron en el diseño del artículo e interpretación de resultados. Todos participaron en la redacción del artículo y aprobaron la versión final.

Conflicto De Intereses: No declarados.

Financiamiento: Autofinanciado.

\section{Como Citar}

Cordova-Vicerrel

Rocio, Huertas-Talavera Eric. Parámetros de calidad de concentrados plaquetarios obtenidos por buffy coat en un hospital general del Perú. Rev méd panacea. 2019; 8(3): 96-100. DOI: https://doi.org/10.35563/rmp.v8i3.192

Recibido: 17 - 08 - 2019

Aceptado: 20 - 09 - 2019

Publicado: 22 - 09 - 2019

\section{RESUMEN}

Objetivo: Verificar si los concentrados plaquetarios obtenidos por buffy coat cumplen con los parámetros de calidad en el banco de sangre de un Hospital General del Perú. Materiales y métodos: Se realizó un estudio descriptivo transversal. La población estuvo constituida por los donantes que acudieron al Banco de sangre del Hospital Regional de lca durante los meses de febrero y marzo del 2017, de donde se obtuvo un tamaño muestral de 67 concentrados plaquetarios que cumplieron con los criterios de inclusión según el PRONAHEBAS, con un nivel de confianza del 95\%, proporción esperada del 5\% y un error del 5\%. Los datos evaluados fueron Inspección visual, presencia de remolino, medición de volumen, medición del potencial de Hidrógeno $(\mathrm{pH})$, recuentos de plaquetas, leucocitos residuales; y a través de cultivos microbiológicos, la contaminación bacteriana, las mismas que fueron llenadas en un Instrumento de evaluación de datos, que luego fueron procesados a través del programa estadístico SPSS vs 22 , aplicándoseles después estadística descriptiva con medidas de frecuencia para las variables cualitativas, y medidas de tendencia central para las variables cuantitativas. Resultados: El 68\% de las muestras evaluadas tuvieron una coloración normal. Sólo el $48 \%$ tuvo un aspecto aceptable con swirling demostrando presencia de plaquetas en forma indirecta. La gran mayoría presentó volúmenes inadecuados de plasma sobrepasando el límite superior. EL pH presentó una amplia gama de variación; sólo la quinta parte de ella tuvo una medida óptima con respecto a este ítem. Los recuentos de plaquetas estuvieron por debajo del recuento aceptable (74\%). La leucorreducción y la presencia de contaminación bacteriana, fueron las características óptimas en un $100 \%$ de la muestra estudiada. Conclusiones: La mayoría de parámetros calidad en el banco de sangre del Hospital Regional de Ica no cumplieron con los requisitos establecidos según estándares establecidos, por lo que el presente estudio sirve de base para continuar con otras investigaciones donde se identifique la falla probable en el procedimiento o patología de nuestros donantes que redunde en una inadecuada obtención de concentrados plaquetarios por el método de buffy coat.

Palabras clave: Parámetros de Calidad, Concentrados plaquetarios, Capa leucoplaquetaria.

\section{ABSTRACT}

Objective: To verify if the platelet concentrates obtained by buffy coat comply with the quality parameters in the blood bank of a General Hospital of Peru. Materials and methods: A cross-sectional descriptive study was carried out. The population was made up of donors who went to the Blood Bank of the Ica Regional Hospital during the months of February and March 2017, where a sample size of 67 platelet concentrates was obtained that met the inclusion criteria according to PRONAHEBAS, with a confidence level of $95 \%$, expected proportion of $5 \%$ and an error of $5 \%$. The data evaluated were visual inspection, swirling presence, volume measurement, hydrogen potential measurement $(\mathrm{pH})$, platelet counts, residual leukocytes; and through microbiological cultures, the bacterial contamination, the same ones that were filled in a Data Evaluation Instrument, which were then processed through the statistical program SPSS vs 22, then applied descriptive statistics with frequency measures for the qualitative variables, and measures of central tendency for quantitative variables. Results: $68 \%$ of the samples evaluated had a normal coloration. Only $48 \%$ had an acceptable appearance with swirling demonstrating the presence of platelets indirectly. The vast majority presented inadequate volumes of plasma exceeding the upper limit. The $\mathrm{pH}$ presented a wide range of variation; Only one fifth of it had an optimal measure with respect to this item. Platelet counts were below the acceptable count $(74 \%)$. Leukoreduction and the presence of bacterial contamination were the optimal characteristics in $100 \%$ of the sample studied. Conclusions: The majority of quality parameters in the blood bank of the Regional Hospital of Ica did not comply the requirements established according to established standards, so the present study serves as a basis to continue with other investigations where the probable failure in the procedure is identified or pathology of our donors that results in an inadequate obtaining of platelet concentrates by the method of buffy coat. 


\section{INTRODUCCIÓN}

Las plaquetas son células sanguíneas fundamentales para la hemostasia (1). Cuando se presentan patologías plaquetarias, ya sea por disminución en el número u otro trastorno en su función, se produce una mala formación del tapón hemostático que recae en una excesiva pérdida de sangre (2). Cuando ocurre esto, es necesaria una transfusión de plaquetas, a excepción de pacientes con trombocitopenia autoinmune o púrpura trombocitopénica trombótica a quienes se les transfunde sólo cuando su vida se encuentra comprometida3. Por lo tanto, la transfusión de plaquetas alogénicas constituye la medida terapéutica más eficaz en el control de las hemorragias de los pacientes con estos defectos (4).

Uno de los métodos más utilizados para la obtención de plaquetas es el fraccionamiento a partir de unidades procedentes de donaciones de sangre total; obteniéndose preferentemente por el método del buffy coat (4). El banco de sangre tipo II del Hospital Regional de Ica, se encarga de estos procedimientos, y por ser un servicio de mayor complejidad y capacidad resolutiva provee unidades de sangre, no sólo a la misma Institución, sino también a los centros de Hemoterapia y Bancos de sangre tipo I (5). Es por ello que se hace necesaria la presente investigación, con el fin de corroborar si se cumplen con los Parámetros de Calidad según el Programa Nacional de Hemoterapia y banco de sangre (PRONAHEBAS), organismo encargado de regular y normar el funcionamiento de los Servicios de Medicina Transfusional en todo el Perú (5), lo cual nos servirá para la corrección y mejora de los procedimientos de obtención de plaquetas en beneficio de los pacientes que lo requieran.

\section{MATERIALES Y MÉTODOS}

Se realizó un estudio transversal de tipo descriptivo, diseño no experimental, observacional. La población en estudio estuvo constituida por los donantes que acudieron al Banco de sangre del Hospital Regional de Ica durante los meses de febrero y marzo del 2017, en donde se aplicó la fórmula para cálculo de muestras de poblaciones finitas, resultando un tamaño muestral de 67 concentrados plaquetarios, con un nivel de confianza del $95 \%$, proporción esperada del 5\% y un error del $5 \%$. Para la selección de la muestra se realizó un muestreo aleatorio simple con reposición de aquellas que cumplieron con los criterios de inclusión establecidos por el Programa Nacional de Hemoterapia y Banco de Sangre (PRONAHEBAS) del Ministerio de salud del Perú. Para la recolección de datos se usó un instrumento de evaluación de datos, en donde se anotaron los resultados de los parámetros de calidad en estudio en el siguiente orden: Inspección visual, presencia de remolino, medición de volumen, medición del potencial de Hidrógeno $(\mathrm{pH})$, recuentos de plaquetas, leucocitos residuales; y a través de cultivos microbiológicos, la contaminación bacteriana. El procesamiento de datos se realizó a través del programa estadístico SPSS VS 22, realizando primero un análisis exploratorio de la base de datos, y luego estadística descriptiva con medidas de frecuencia para las variables cualitativas, y medidas de tendencia central para las variables cuantitativas.

\section{RESULTADOS}

Se evaluaron 67 concentrados plaquetarios. En la inspección visual de valoró el color. La mayor parte de los concentrados plaquetarios presentaron una coloración amarillenta normal (68\%), y un $20 \%$ de sueros lipémicos. No se encontró ninguna unidad con tonalidad ictérica.

\section{Característica Física: COLOR}

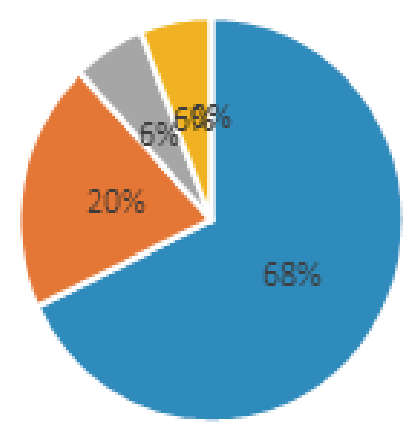

\section{- 1 Amarillo \\ - 2 Lipémico \\ - 3 Verdoso \\ - 5 Hemolizado}

Figura 1: Característica física: COLOR de los concentrados plaquetarios. FUENTE: Elaboración propia sobre la base de la ficha de evaluación.

En relación al aspecto, evaluado por la presencia de remolino, la mayor parte de la muestra estudiada (58\%) no presentó swirling o remolinos de plaquetas.

\section{Característica Física: ASPECTO}

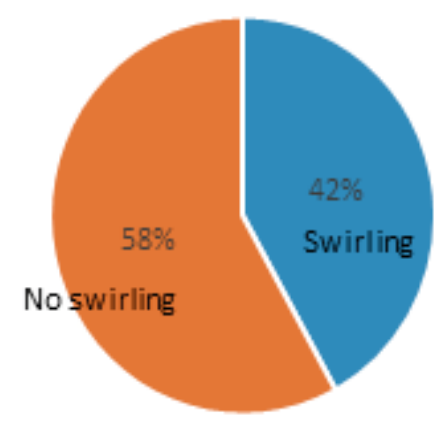

Figura 2: Característica física: ASPECTO de los concentrados plaquetarios. FUENTE: Elaboración propia sobre la base de la ficha de evaluación.

Con respecto al volumen, sólo el $12 \%$ de unidades de concentrados plaquetarios presentaron un volumen adecuado, notándose un gran porcentaje de concentrados plaquetarios con volúmenes aumentados 
(84\%).

En la tabla $\mathrm{N}^{\circ} 01$ se puede ver las frecuencias relativas del $\mathrm{pH}$, volumen, recuento de plaquetas y recuento de glóbulos blancos. Con respecto al $\mathrm{pH}$, se observó que sólo un $22 \%$ del total de muestras evaluadas cumplieron con los parámetros normales, presentado un amplio rango de valores que oscilaron entre 5.9 a $8.2 \mathrm{de} \mathrm{pH}$ tal como se muestra en el gráfico 1.

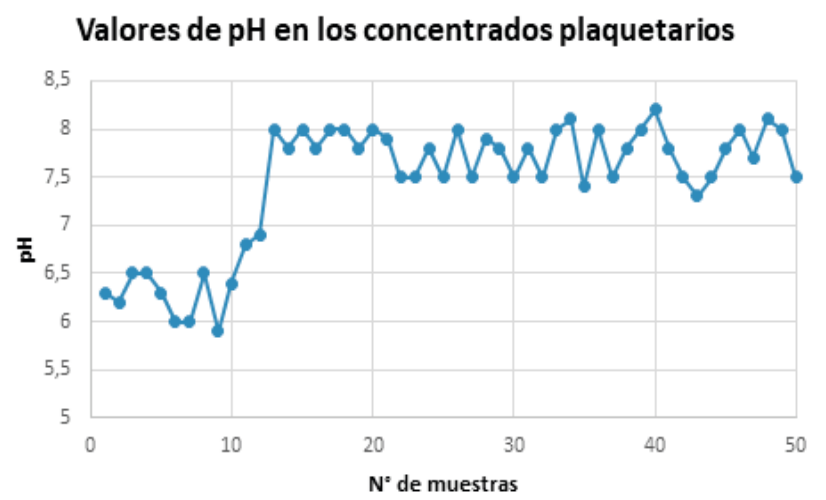

Grafico 1: $\mathrm{pH}$ de los concentrados plaquetarios. FUENTE: Elaboración propia sobre la base de la ficha de evaluación.

Así también, sólo el $26 \%$ de las unidades de concentrados plaquetarios cumplieron con un recuento óptimo de plaquetas; y en relación a la presencia de leucocitos residuales en dichos productos, el $100 \%$ de ellas estaban leucorreducidas, no excediendo ninguna el parámetro normal.

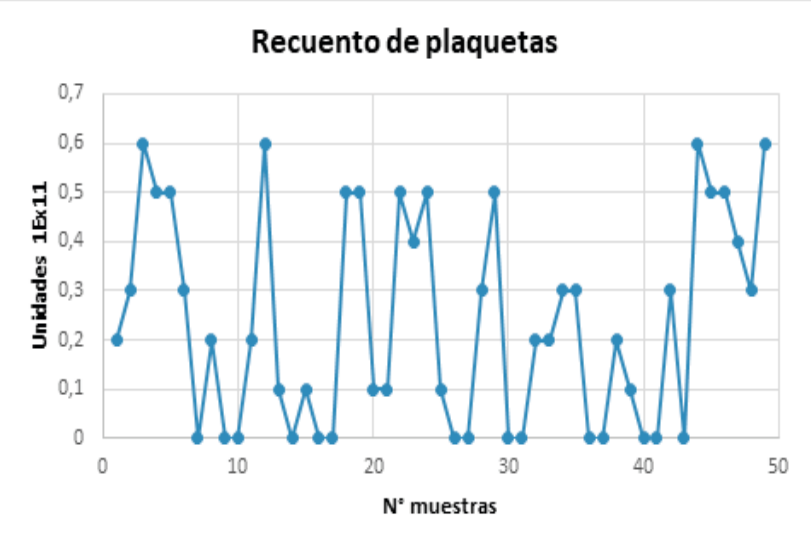

Grafico 2: Número de plaquetas contenidas en los concentrados plaquetarios. FUENTE: Elaboración propia sobre la base de la ficha de evaluación.

Referente a la Contaminación bacteriana, la totalidad de las muestras evaluadas estaban libres de contaminación.

\section{Contaminación bacteriana}

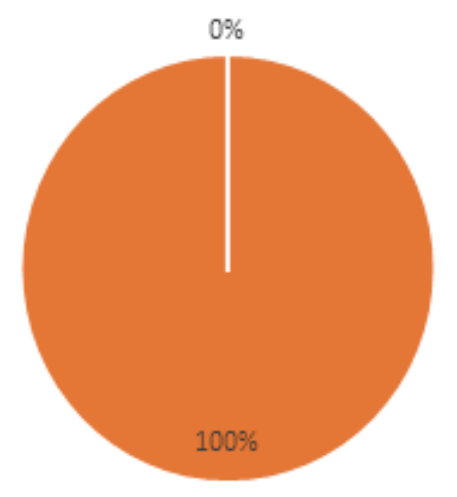

- 1 Presencia

$=2$ Ausencia

Figura 3: Característica física: ASPECTO de los concentrados plaquetarios. FUENTE: Elaboración propia sobre la base de la ficha de evaluación.

Con respecto al análisis bivariado, se aplicó la correlación de Pearson buscando relación entre las variables cuantitativas para establecer dependencia entre ellas. La relación volumen y recuento de plaquetas, sólo presentó una dependencia del $1 \%$ y en la relación volumen y presencia de leucocitos residuales tampoco se halló correspondencia, por lo que este tipo de análisis no pudo ser llevado a cabo.

\section{DISCUSIÓN}

El control de calidad de los concentrados plaquetarios se basa en realizar actividades técnicas en forma periódica, que aseguren el cumplimiento de los estándares establecidos para la producción de componentes sanguíneos en los bancos de sangre o centros de medicina transfusional (6). Es por ello que con el fin de conocer los productos que estamos ofreciendo a los pacientes del hospital regional de lca $u$ otros establecimientos de salud del cual dependen, se hizo necesario evaluar los estándares de calidad de los concentrados plaquetarios.

En el presente trabajo se evaluaron las características físicas y de calidad de los concentrados plaquetarios. La coloración presentada fue en su mayor parte normal, presentándose un $68 \%$ de las mismas con coloración amarillenta. Un buen porcentaje de estas plaquetas presentaron un color lipémico, verdoso y hemolizado (32\%). Este último podría deberse a un defecto en el proceso de fraccionamiento, el cual, al romperse el glóbulo rojo, éste libera la hemoglobina contenida en su interior, transmitiendo un color rojizo al producto final6. Los colores lipémico y verdoso son debidas a patologías del donante7. Dichas patrologías pueden presentarse en los donantes debido a que en los exámenes preliminares del donante, se incluyen hemoglobina y hematocrito y grupo sanguíneo, más no se incluyen los perfiles hepáticos o lipídicos que nos permitirían obtener una mayor apreciación de patologías del donante.

Otra característica evaluada, relacionada con la presencia 
Parámetros de calidad de concentrados plaquetarios obtenidos por buffy coat en un Hospital

General del Perú.

macroscópica de plaquetas fue el aspecto, es decir la presencia o no de swirling. Esto se refiere a la presencia de cúmulos plaquetarios suspendidos en un volumen de plasma (7). En el presente estudio, sólo un $42 \%$ de las muestras estudiadas presentaron swirling, lo que significa que la mayor parte de concentrados plaquetarios no cumplieron con este parámetro de calidad. La evaluación del aspecto, pues es una forma de ver indirectamente la presencia o no de plaquetas y tiene una relación directamente proporcional con el recuento total de plaquetas de cada unidad de concentrado plaquetario (8).

El volumen fue otra característica evaluada que no cumplió con el estándar de calidad, ya que la mayor parte de ellas presentó más volumen de lo normal (84\%). Esta característica del volumen, es importante en pacientes con patologías subyacentes, como por ejemplo en insuficiencia cardiaca congestiva, ya que incurriría en una sobrecarga de líquidos que pudiera ocasionar una complicación frecuente en estos pacientes como insuficiencia respiratoria aguda por edema agudo de pulmón9. Sin embargo, en su estudio Saritama obtuvo un porcentaje de 63.3\%, 91.70\%, 97.50\% y $97.50 \%$ en los parámetros evaluados como recuento plaquetario, recuento leucocitario, volumen del concentrado plaquetario y el $\mathrm{pH}$, respectivamente (10). Esta inadecuada proporción de volumen puede ser explicada en el fraccionamiento manual, cuando se utiliza fraccionadores manuales y no semiautomáticos o automáticos, como ocurrió en la presente investigación. Los fraccionadores semiautomáticos estandarizan el volumen de plasma en el que deben ir las plaquetas para que puedan cumplir con los parámetros establecidos(10).

Se observó una amplia variedad de $\mathrm{pH}$ (desde 5.9 a 8.2), presentando la mayor parte de ellas un valor fuera del rango normal (6.4 a 7.4). Este factor evaluado constituye un buen marcador para la viabilidad de las plaquetas contenidas dentro del concentrado plaquetario ya que su aumento o disminución están relacionadas con una contaminación bacteriana o por un exceso de glucólisis dentro de las lesiones por almacenamiento, respectivamente6. Además una proporción de sangre disminuida con respecto al anticoagulante durante la extracción sanguínea, puede ocasionar también una alteración en el $\mathrm{pH}(11,12)$.

Asimismo, en relación al recuento plaquetario, sólo el $26 \%$ cumplió con el recuento de plaquetas requerido. En un estudio similar, Njoroge(13) demostró un estudio en el que sólo el $51 \%$ de todas las concentraciones plaquetarias cumplían con el requisito mínimo del recuento de plaquetas $>5.5 \times 1010$. Esto puede darse por varios motivos. Uno de ellos es por los tiempos inadecuados de reposo del Buffy coat durante el proceso(14) otro, es porque al momento de calificar al donante no se hace un recuento previo de sus plaquetas(6), y otra causa pudo ser al aumento de volumen de plasma, la misma que diluye los concentrados plaquetarios(15), disminuyendo el contaje final.

Los Glóbulos blancos transfundidos es una de las causas más frecuentes de reacción transfusional(6), es por ello que se requiere concentrados de plaquetas leucorreducidas, lo que significa que en una unidad debe haber menos de un millón de leucocitos. En el presente análisis no se observaron unidades con cantidades superiores a las permitidas cumpliendo en un $100 \%$ con este parámetro de calidad.

Las plaquetas, son los únicos componentes sanguíneos que se deben conservar a temperatura ambiente para una sobrevida de 5 días (17), luego de ese tiempo ya no son viables funcionalmente. Debido a esta característica, dichos concentrados plaquetarios son muy susceptibles de contaminarse, es por ello que se realizó el cultivo microbiológico, para ver crecimiento de unidades formadoras de colonias de bacterias. A las 48 horas de la siembra y posterior incubación, se observó que la totalidad de muestras analizadas estaban libres de contaminación bacteriana, al igual que el estudio de Pineda (6) donde sus cultivos también estuvieron exento de contaminación. Esto traduce las buenas prácticas de asepsia y antisepsia de la zona de punción antes de la extracción de sangre total en los donantes que acuden al banco de sangre del Hospital Regional de Ica. Sin embargo en un estudio en Zinbabwe (18), se encontró un alto porcentaje de contaminación en sus unidades de concentrados plaquetarios, aduciendo probablemente medidas asépticas inadecuadas.

Tanto los volúmenes, el recuento de plaquetas y el aspecto con ausencia de swirling fueron las características que no cumplieron con los criterios de calidad; el escaso personal capacitado, la falta de exámenes más rigurosos en el donante, y quizás la falta de equipos para la obtención de estos productos, podrían ser las causales del no cumplimiento a cabalidad de estas características. En un estudio comparativo en la obtención de plaquetas por buffy coat y aféresis, si bien los concentrados plaquetarios por el método de buffy coat contaban con menor cantidad de remolinos, volumen y $\mathrm{pH}$ se encontró que aun así estaban dentro de los límites de control de calidad requeridos; esto es importante puesto que los concentrados plaquetarios bien procesados por el método de buffy coat pueden usarse de manera efectiva en la mayoría de los pacientes con trombocitopenia en un entorno de pacientes con pocos recursos (19). Quizá el tiempo de reposo de la sangre entera después de la extracción influya mucho en la mejora de las características finales de los concentrados plaquetarios (20).

No se presentaron relaciones de dependencia entre una y otra característica, por lo que no se pudo llevar a cabo el análisis bivariado en el presente trabajo. 


\section{REFERENCIAS BIBLIOGRÁFICAS}

1. Gómez-Gómez B, Rodríguez-Weber FL, Díaz Greene EJ. Fisiología plaquetaria, agregometría plaquetaria y utilidad clínica. Med Int Méx. 2018; 34(2):244-263

2. Martinuzzo M. Sistema de coagulación. Blood Coagulation System Physiology. Hematología. 2017; 21(1): 31-42

3. Vicente - Llau, J. y Col. Medicina transfusional perioperatoria. $2^{\circ}$ edición. Editorial Elsevier. 2019

4. Ramos-Quiroz, M. y Col. Comparación entre los rendimientos arrojados por el equipo Trima Accel y los rendimientos de control de calidad para aféresis plaquetarias en el banco de sangre del Hospital Infantil Teletón de Oncología. 2017;10(1):3-49

5. Ministerio de Salud. Gobierno del Perú. Lineamientos de Política del PRONAHEBAS Lima: Dirección General De Salud de las Personas. Dirección de Servicio de Salud. Programa Nacional de Hemoterapia y Bancos de Sangre [Internet]. Citado el 8 de julio del 2019. Disponible en: http://bvs.minsa.gob.pe/local/MINSA/1087_DGSP264 .pdf

6. Pineda, GS. Evaluación de la calidad de concentrados plaquetarios obtenidos a partir de sangre total en el Hemocentro de la Cruz Roja Ecuatoriana 2014-2015. Pontificia Universidad Católica del Ecuador; 2015.

7. American Association of Blood Banks. (2017). AABB $18^{\circ}$ edición. Buenos Aires: Asociación Argentina de Hemoterapia e Inmunohematología.

8. Arenas, F. Correlación de los Concentrados plaquetarios con las condiciones de control de Hemocomponentes en el Servicio de Hemoterapia del Hospital Base Case ESSALUD. Arequipa-2015 [Tesis de Grado]. Universidad Alas Peruanas. Perú. 2015

9. Hernández-López G, Zamora. S, Gorordo L, García-Román M, Jiménez-Ruiz A, Tercero B. Síndrome de Insuficiencia Respiratoria Aguda. Rev Hosp Jua Mex 2015;82(1): 31-42

10. Saritama Celi L. Control de Calidad de Concentrados Plaquetarios almacenados en el Servicio de Medicina Transfusional del Hospital Pediátrico Baca Ortiz Mediante la Medición de Volumen, Potencial de Hidrógeno, Recuento Plaquetario y Recuento Leucocitario Residual. [Tesis de grado]. Quito: Facultad de Ciencias Médicas-Carrera Laboratorio Clínico e Histotecnólogico. Universidad Central del Ecuador; 2016. 86 pp.

11. Álvarez Trujillo T. Cueva Tolentino M. Parámetros de Calidad de los concentrados plaquetarios obtenidos por capa leucoplaquetaria en el Hospital Nacional Alberto Sabogal Sologuren, 2018. [Tesis para la Obtención de Título de Especialista en Hemoterapia y Banco de Sangre]. Lima: Facultad de Ciencias de la Salud-Escuela Académico Profesional de Tecnología Médica, Universidad Norbert Wiener; 2018

12. Gal B, López M, Martín A. Prieto J. Bases de la Fisiología. Segunda Edición. Madrid. Editorial Tébar; 2007. 85 pp.
12. Njoroge NR, maturi PM, Githanga J, Jamilla R. Quality parameters of platelet concentrate at Kenyatta National Hospital's Blood Transfusion Unit.

13. International journal of Hematological Disorders. 2014;1(1): 35-40.

Zaragozá, C. (2012). Análisis de las interacciones de polifenoles con el receptor GPIIB/IIla plaquetario efectos inmunomoduladores sobre células

14. sanguíneas circulantes. Tesis:Universidad de Alcala-Departamento de farmacología, 29-34. Disponible en: http://dspace.uah.es/dspace/bitstream/handle/1001 7/15901/TESIS\%20Cristina\% 0Zaragoz\%C3\%A1.pdf?sequence $=1$

Puig, L. Fraccionamiento primario de la sangre y conservación de productos sanguíneos. Medicina transfusional y terapia celular. 2010(1)1-50.

15. Norma Técnica Peruana N¹4-MINSA/DGSP. Sistema de Gestión de la Calidad del PRONAHEBAS. Guía de Procedimientos Operativos Estándar.

16. Ministerio de Salud. Lima-Perú. 2004. Vol 01 Tromboflebitis. (2013). Tromboflebitis.net. Recuperado el 4 de Mayo de 2015, de Trombocitopenia. Disponible en:

17. http://tromboflebitis.net/trombocitopenia/ Ngonidzashe, M. Clifford, S. y Rooyen, M. Prevalencia de contaminación bacteriana en sangre y derivados en el servicio de banco de sangre de

18. zimbabwe. 2015: 12(1): 18-25

Mallhi RS, Kumar S, Philip J. "Evaluación comparativa de la calidad de los concentrados de plaquetas preparada por el método de Buffy Coat y el método

19. de aféresis". Indian Journal of Hematology and Blood Transfusion. [Internet] 2015. [Citado el 26 de marzo del 2019] Vol 31: 453-459. Disponible en: https://doi.org/10.1007/s12288-014-0476-z

Díaz Yuto A. Tiempo de sedimentación y parámetros de calidad en concentrados plaquetarios del Hospital Nacional Hipólito Hunánue 2017. [Tesis

20. para la obtención de Título de especialista en Hemoterapia y Banco de Sangre]. Lima: Sección de post grado- Segunda especialidad en Hemoterapia y Banco de Sangre de la Facultad de Tecnología Médica. Universidad Nacional Federico Villarreal; 2018.

\section{PANACEA}

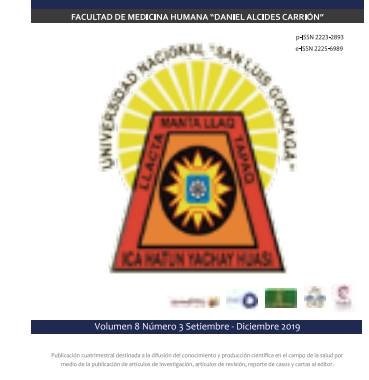

